03,08

\title{
Особенности высокочастотной ЭПР/ЭСЭ/ОДМР спектроскопии NV-дефектов в алмазе
}

\author{
( Р.А. Бабунц, Д.Д. Крамущенко, А.С. Гурин, А.П. Бундакова, М.В. Музафарова, \\ А.Г. Бадалян, Н.Г. Романов, П.Г. Баранов
}

Физико-технический институт им. А.Ф. Иофрфе РАН, Санкт-Петербург, Россия

E-mail: roman.babunts@gmail.com

Поступила в Редакцию 20 июня 2020 г.

В окончательной редакции 20 июня 2020 г.

Принята к печати 30 июня 2020 г.

Методы высокочастотного электронного парамагнитного резонанса (ЭПР), электронного спинового эха (ЭСЭ) и оптически детектируемого магнитного резонанса (ОДМР) использованы для исследования уникальных свойств азотно-вакансионных дефектов (nitrogen-vacancy NV center) в алмазе в сильных магнитных полях. Показано, что в сильных магнитных полях $(\sim 3-5 \mathrm{~T})$ происходит эффективное оптически индуцированное выстраивание населенностей спиновых уровней, с заполнением уровня $M_{S}=0$ и опустошением уровней $M_{S}= \pm 1$, что позволило регистрировать ОДМР по изменению интенсивности фотолюминесценции, достигающим 10\% в момент резонанса. Продемонстрировано, что эта эффективность имеет тот же порядок, что и в нулевых и низких магнитных полях. Образцы предварительно исследовались методом ОДМР в нулевых магнитных полях, что позволило точно определить основные параметры тонкой структуры и сверхтонкие взаимодействия с ядрами азота, а также диполь-дипольные взаимодействия между NV-центром и глубокими донорами азота в виде атома азота, замещающего углерод, $\mathrm{N}^{0}$. В спектрах высокочастотного ОДМР наблюдались сверхтонкие взаимодействия с ближайшими атомами углерода (изотоп $\left.{ }^{13} \mathrm{C}\right)$, что открывает возможности для измерения оптическими методами процессов динамической поляризации ядер углерода в сильных магнитных полях. Предполагается, что узкие линии ОДМР в сильных магнитных полях могут быть использованы для измерения этих полей с субмикронным пространственным разрешением. Разработан новый метод регистрации ОДMP NV-центров с модуляцией микроволновой частоты, упрощающий технику измерения высоких магнитных полей. Продемонстрировано значительное увеличение интенсивности сигнала ОДМР при ориентации сильного магнитного поля вдоль оси симметрии NV-центра.

Ключевые слова: электронный парамагнитный резонанс, оптически детектируемый магнитный резонанс, спектрометр ЭПР, алмаз, NV-дефект.

DOI: 10.21883/FTT.2020.11.50101.137

\section{1. Введение}

Азотно-вакансионный (nitrogen-vacancy, NV) дефект в алмазе представляет собой пару из атома азота и вакансии углерода, при этом, азот замещает один из четырех ближайших к вакансии атомов углерода [1]. NV-центр в алмазе существует в двух зарядовых состояниях: нейтральном зарядовом состоянии $\mathrm{NV}^{0}$ со спином $S=1 / 2$ и отрицательно-заряженном состоянии $\mathrm{NV}^{-}$со спином $S=1 . \mathrm{NV}^{-}$-центр в алмазе является одним из наиболее изученных дефектов в полупроводниках и наиболее важным дефектом для применений, что обусловлено существованием уникального механизма оптически индуцированного выстраивания населенностей спиновых уровней в широком диапазоне температур, вплоть до комнатной температуры и выше. $\mathrm{Ha} \mathrm{NV}^{-}$-центре в алмазе была впервые реализована схема по манипуляции спинами одиночного центра при комнатной температуре [2-4]. Эти фундаментальные свойства были использованы для создания нового поколения квантовых сенсоров магнитного поля [5-7], включая биологические сенсоры [8-10] с субмикронным простраственным разрешением, источников одиночных фотонов [11], элементной базы для квантовых компьютеров [11]. Большие возможности для биологических применений открываются при использовании наночастиц алмаза, содержащих $\mathrm{NV}^{-}$-центры $[6,9,12,13]$.

Эффективность процессов оптически индуцированного выстраивания населенностей спиновых уровней доказана многочисленными исследованиями оптически детектируемого магнитного резонанса (ОДМР) в нулевом, малых и средних магнитных полях, однако нет работ по исследованию ОДМР в сильных магнитных полях и при низких температурах. При этом не очевидно, что в этих условиях ОДМР будет эффективен. Одной из задач настоящей работы было выполнение таких исследований. Исследовались также спектры высокочастотного электронного парамагнитного резонанса (ЭПР) и электронного спинового эха (ЭСЭ).

\section{2. Методика эксперимента}

Исследовались монокристаллы искусственных алмазов, выращенных при высоких давлениях и температу- 


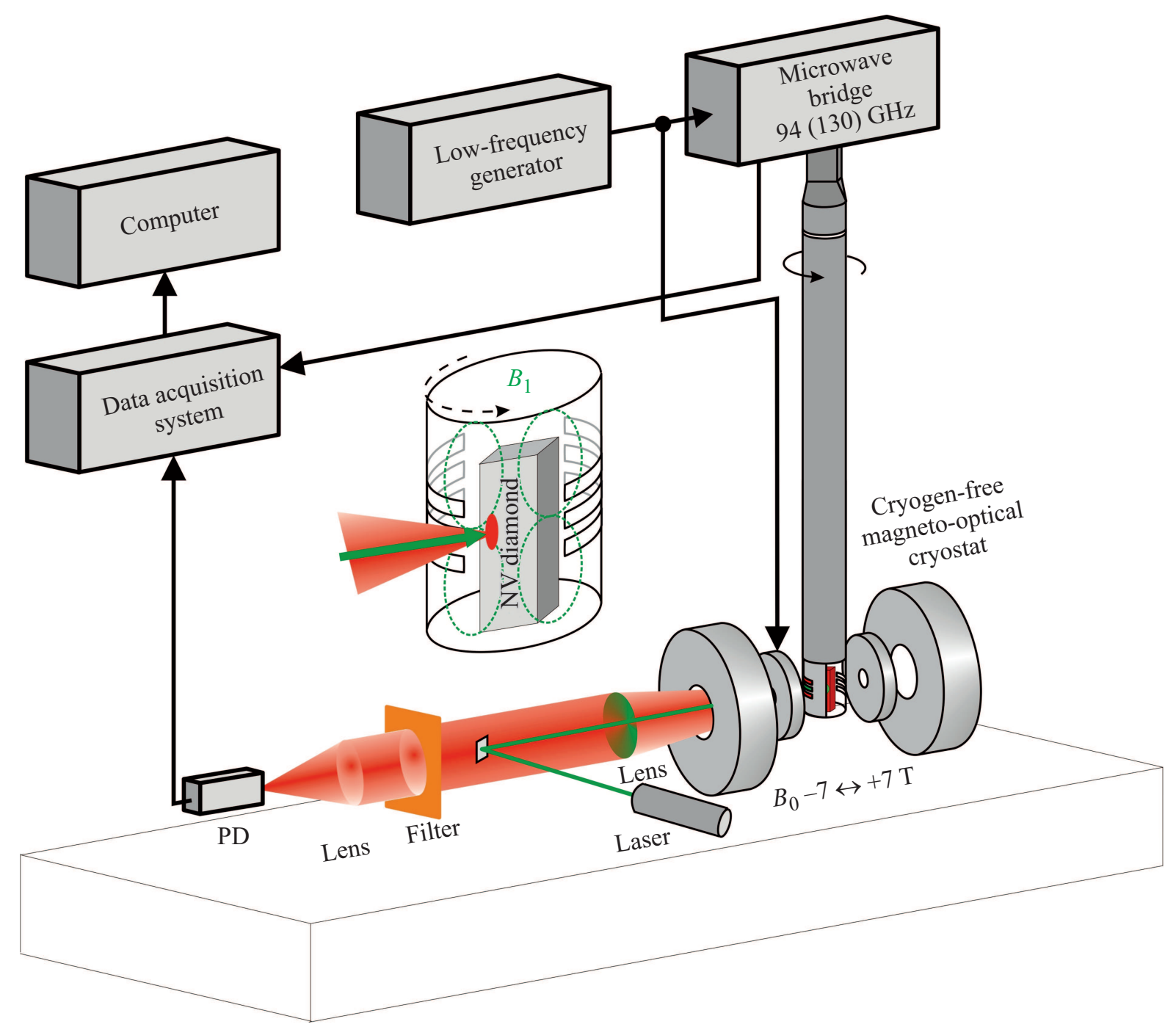

Рис. 1. Упрощенная схема спектрометра ЭПР/ЭСЭ/ОДМР, работающего в высокочастотных $130 \mathrm{GHz}(2 \mathrm{~mm})$ и $94 \mathrm{GHz}(3 \mathrm{~mm})$ диапазонах в сильных магнитных полях. На вставке в увеличенном масштабе приведена конструкция оптического возбуждения и регистрации ФЛ в образце, помещенном в микроволновый стакан с прорезью для оптического возбуждения, который используется в диапазонах 2 и $3 \mathrm{~mm}$, т. е. эта конструкция может быть применена при перестройке длины волны в области $2-3 \mathrm{~mm}$.

pax (high-pressure high-temperature growth). Концентрация азота в них составляла $\sim 50 \mathrm{ppm}$. Образцы были облучены быстрыми нейтронами в реакторе с дозой $\sim 10^{18} \mathrm{~cm}^{-2}$ с последующим отжигом $(\sim 1 \mathrm{~h})$ в водороде при температуре $800^{\circ} \mathrm{C}$. В результате концентрация $\mathrm{NV}$-центров составляла примерно 2-3 ppm. Образцы контролировались оптическими методами, а также методом ОДМР в нулевом и малых магнитных полях на частотах в диапазоне $2.5-3 \mathrm{GHz}$ и в широком диапазоне температур, включая комнатные и выше комнатной температуры.

Образцы имели размеры $\sim 3 \times 3 \times 1 \mathrm{~mm}$ и устанавливались таким образом, чтобы можно было вращать их вокруг ребра, совпадащего с одним из направлений $\langle 110\rangle$. Отклонение оси вращения от $\langle 110\rangle$ составляло несколько градусов. В работе использовался многоцелевой высокочастотный спектрометр
ЭПР/ЭСЭ/ОДМР [23]. На рис. 1 представлена упрощенная схема спектрометра, работающего в высокочастотных $130 \mathrm{GHz}(2 \mathrm{~mm})$ и $94 \mathrm{GHz}(3 \mathrm{~mm})$ диапазонах в сильных магнитных полях, созданного в ФТИ им. А.Ф. Иоффе в кооперации с компанией ДОК $[14,15,16]$. На вставке в увеличенном масштабе показана схема оптического возбуждения и регистрации фотолюминесценции (ФЛ) образца, помещенного в закороченный круглый волновод с прорезями для прохождения света возбуждения и ФЛ. Эта конструкция может быть применена при перестройке длины волны в области 2-3 mm.

\section{3. Экспериментальные результаты и обсуждение результатов}

Образцы предварительно исследовались методом ОДМР в нулевых магнитных полях, что позволило 
определить основные параметры тонкой структуры и сверхтонких взаимодействий с ядрами азота, а также диполь-дипольные взаимодействий между NV-центром и глубокими донорами азота $\mathrm{N}^{0}$.

На рис. 2 представлены спектры ОДМР NV-центров и пар $\mathrm{NV}-\mathrm{N}^{0}$ в нулевом магнитном поле, зарегистрированные при температурах 25 и $260^{\circ} \mathrm{C}$ в облученном быстрыми нейтронами и отожженом при $800^{\circ} \mathrm{C}$ монокристалле алмаза. Спектры ЭПР NV-центров описываются спиновым гамильтонианом

$$
\begin{aligned}
H & =g_{e} \mu_{\mathrm{B}} \mathbf{B} \cdot \mathbf{S}+D\left[\mathbf{S}_{\mathbf{z}}^{2}+S(S+1)\right]+E\left(\mathbf{S}_{\mathbf{x}}^{2}-\mathbf{S}_{\mathbf{y}}^{2}\right) \\
& +\mathbf{S} \cdot \mathbf{A}_{\mathrm{N}} \cdot \mathbf{I}_{\mathrm{N}}+\mathbf{I}_{\mathrm{N}} \cdot \mathbf{P}_{\mathrm{N}} \cdot \mathbf{I}_{\mathrm{N}}+\sum_{i} \mathbf{S} \cdot \mathbf{A}_{\mathrm{C} 13 \mathrm{i}} \cdot \mathbf{I}_{\mathrm{C} 13 \mathrm{i}} .
\end{aligned}
$$

Все члены спинового гамильтониана имеют стандартные обозначения. (i) Первое слагаемое представляет собой зеемановское взаимодействие в магнитном поле В, произведение электронного $g$-фактора NV-центра $\left(g_{e}=2.003\right)$ на магнетон Бора $\mu_{\mathrm{B}}$ является гиромагнитным отношением $\gamma=g \mu_{\mathrm{B}}$. (ii) Второе и третье слагаемые отражают тонкую структуру, где параметры $D=2.87 \mathrm{GHz}$ и $E=3 \mathrm{GHz}$ (при $T=25^{\circ} \mathrm{C}$ ) соответствуют аксиальной и поперечной составляющим этого взаимодействия. (iii) Четвертое и пятое слагаемые описывают сверхтонкое взаимодействие (СТВ) с атомом азота (изотоп ${ }^{14} \mathrm{~N}, I=1$ ) с анизотропной константой СТВ $A_{\|}=-2.14 \mathrm{MHz}, A_{\perp}=-2.70 \mathrm{MHz}$ и квадрупольное взаимодействие с константой квадрупольного взаимодействия $P_{\|}=-5.01 \mathrm{MHz}$, которое аксиально симметрично относительно оси симметрии центра $C_{3 v}$ [17]; (vi) шестое слагаемое отражает анизотропное СТВ с одним ядром ${ }^{13} \mathrm{C}$, находящемся в положении одного из трех атомов углерода в ближайшем окружении вакансии. Зеемановские взаимодействия ядер азота и ядер углерода ${ }^{13} \mathrm{C}$ с магнитным полем опущены в спиновом гамильтониане, так как они примерно на три порядка меньше зеемановского взаимодействия для электронов и практически не проявляются в спектрах ЭПР, однако чрезвычайно важны в экспериментах по двойному электронно-ядерному резонансу (ДЭЯР). Приведенные на рис. 2 спектры ОДМР описываются спиновым гамильтонианом (1) в условиях нулевого магнитного поля $B=0$ и позволили нам с большой точностью определить параметры тонкой и сверхтонкой структуры.

Центральная интенсивная линия соответствует расщеплению тонкой структуры $D$, дополнительное расщепление этой линии обусловлено напряжениями в кристалле и равно $2 E$. Верхние вставки демонстрируют представленные в увеличенном масштабе низкополевой и высокополевой сателлиты для спектра, зарегистрированного при $25^{\circ} \mathrm{C}$. Видна сверхтонкая структура, соответствующая по величине сверхтонкому взаимодействию с ядром азота в NV-центре. Расщепление сателлитов, показанное вверху стрелкой, по величине примерно соответствует сверхтонкому взаимодействию с ядром азота ${ }^{14} \mathrm{~N}$ в $\mathrm{N}^{0}$-центре. Таким образом, сверхтонкие переходы внутри $\mathrm{N}^{0}$-центра влияют на интенсивность фотолюминесценции NV-центра, в то время как сверхтонкие переходы внутри NV-центра воздействуют на структуру сателлитов. Свидетельства прямого и обратного переноса сверхтонкого взаимодействия с ядрами азота в связанных $\mathrm{NV}-\mathrm{N}$ парах в алмазе ранее было продемонстрировано в нашей работе [18]. Показан значительный температурный сдвиг расщепления тонкой структуры $D[18,19]$, который нашел широкое применение при локальном измерении температур с наноразмерным разрешением [20]. На нижней правой вставке проведено сравнение неаксиального расщепления $2 E$ при комнатной $\left(25^{\circ} \mathrm{C}\right)$ и высокой $\left(260^{\circ} \mathrm{C}\right)$ температурах, в относительной шкале частот. Видно, что параметр $E$ уменьшается при увеличении температуры, и линии сужаются, что, по-видимому, обусловлено процессами динамического усреднения напряжений в кристалле вблизи NV-центра, а также усреднению сверхтонких взаимодействий с азотом, входящим в структуру NV-центра.

На нижней левой вставке рис. 2 представлена схема, описывающая цикл оптического возбуждения и излучения $\mathrm{NV}$-центра, где ${ }^{3} A$ и ${ }^{3} E$ являются триплетным основным и возбужденным состояниями, ${ }^{1} A$ и ${ }^{1} E$ являются метастабильными состояниями, ISC (inter-system crossing) представляет собой межсистемные переходы в метастабильное состояние. Разрешенные оптические переходы между основным и возбужденным состояниями показаны сплошными линиями, безызлучательные переходы показаны пунктирными линиями. Толщина стрелок условно обозначает скорости перехода между различными состояниями. В зависимости от скорости переходов система релаксирует либо посредством флуоресцентного излучения, либо безызлучательно через ISC в метастабильное состояние. Вследствие разных скоростей переходов сразу после включения возбуждения $(532 \mathrm{~nm})$ в течение короткого времени (менее $1 \mu \mathrm{s}$ ) система населенностей спиновых уровней выстраивается в виде заселения уровня $M_{S}=0$ и опустошения уровней $M_{S}= \pm 1$, условно показанные количеством шаров (рис. 2). Это приводит к отключению безызлучательного канала и увеличению интенсивности ФЛ более чем на 10\%. При приложении резонансного микроволнового излучения происходит выравнивание населенностей спиновых уровней в основном состоянии, что приводит снова к включению безызлучательного канала и уменьшению интенсивности ФЛ, то есть к оптическому детектированию магнитного резонанса. В работе [18] показано, что интенсивности сигналов ОДМР существенно увеличиваются при помещении алмаза с NV-центрами в жидкость, например в воду, что обусловлено диэлектрическими свойствами жидкости. Эти исследования открывают возможности по использованию метаматериалов для повышения эффективности сбора ФЛ NV-центров в алмазе.

На рис. 3 показана структура единичной ячейки алмаза с изображением отрицательно заряженного $\mathrm{NV}$-центра со спином $S=1$ и удаленного глубокого донора азота $\mathrm{N}^{0}$ со спином $S=1 / 2$, представлящего собой атом азота, замещающий углерод. Стрелками 


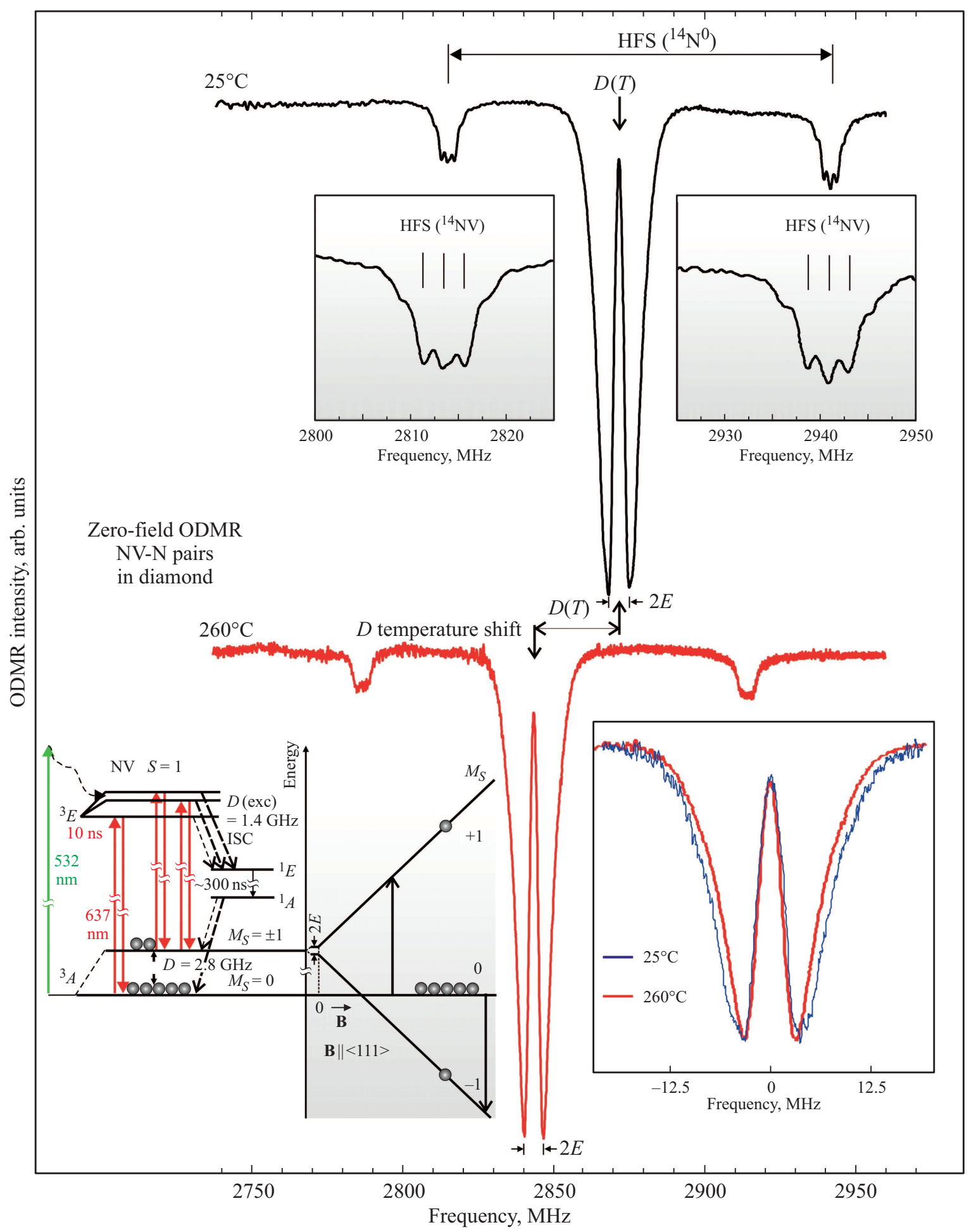

Рис. 2. Спектры ОДМР $\mathrm{NV}$-центров и $\mathrm{NV}-\mathrm{N}^{0}$ пар в нулевом магнитном поле, зарегистрированные при температурах 25 и $260^{\circ} \mathrm{C}$ в облученном быстрыми нейтронами и отожженом при $800^{\circ} \mathrm{C}$ монокристалле алмаза. Верхние вставки демонстрируют представленные в увеличенном масштабе низкополевой и высокополевой сателлиты для спектра, зарегистрированного при $25^{\circ} \mathrm{C}$. На нижней левой вставке представлена схема, описывающая цикл оптического возбуждения и излучения $N V$-центра, где ${ }^{3} A$ и ${ }^{3} E$ являются триплетным основным и возбужденным состояниями, ${ }^{1} A$ и ${ }^{1} E$ являются метастабильными состояниями, ISC представляет собой межсистемные переходы (inter-system crossing - ISC) в метастабильное состояние. Разрешенные оптические переходы между основным состоянием и возбужденным состоянием показаны сплошными линиями, безызлучательные переходы показаны пунктирными линиями. Толщина стрелок условно обозначает скорости перехода между различными состояниями.

На нижней правой вставке проведено сравнение неаксиального ращепления $2 \mathrm{E}$ при комнатной и высокой температурах, при этом шкала частот соответствует сигналу при $260^{\circ} \mathrm{C}$, масштаб одинаковый для двух температур. Показан также значительный температурный сдвиг расщепления тонкой структуры $D(T)$. 


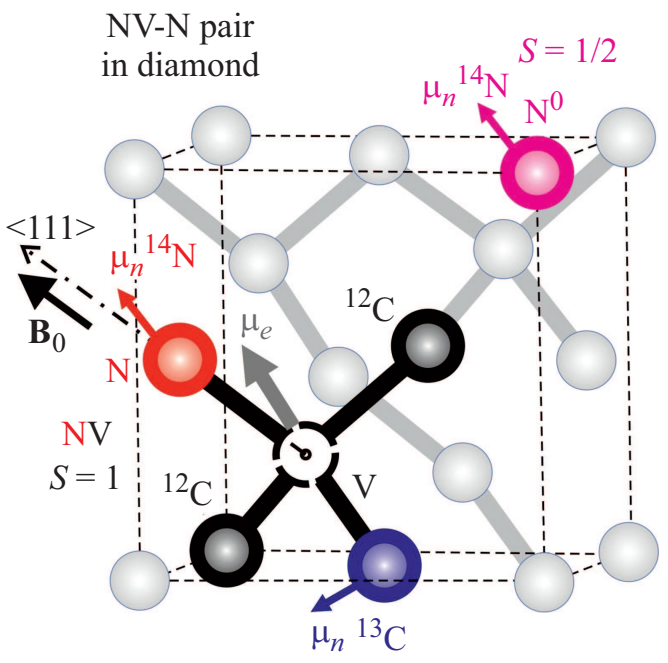

Pис. 3. Структура единичной ячейки алмаза с изображением отрицательно заряженного NV-центра со спином $S=1$ и удаленного глубокого донора азота $\mathrm{N}^{0}$ со спином $S=1 / 2$. Стрелками условно показаны магнитные моменты изотопов азота $\left({ }^{14} \mathrm{~N}\right)$ и углерода $\left({ }^{13} \mathrm{C}\right)$, имеющих ядерные магнитные моменты, при этом выбрана ситуация, когда из трех атомов углерода один атом является изотопом ${ }^{13} \mathrm{C}$ с ядерным магнитным моментом. условно показаны магнитные моменты изотопов азота $\left({ }^{14} \mathrm{~N}\right)$ и углерода $\left({ }^{13} \mathrm{C}\right)$, имеющих ядерные магнитные моменты, при этом выбрана ситуация, когда из трех атомов углерода один атом является изотопом ${ }^{13} \mathrm{C}$ с ядерным магнитным моментом [21]. Изотоп азота ${ }^{15} \mathrm{~N}$ не рассматривался ввиду его очень низкого содержания.

Средние расстояния между дефектами в алмазе непосредственно связаны с их концентрациями. Типичные концентрации NV-центров и замещающих атомов азота $\mathrm{N}^{0}$ порядка единиц и десятков ppm, соответственно. Согласно работе [22], среднее расстояние оценивается из предположения, что вероятность отсутствия ближайшего дефекта внутри расстояния $r$ от NV-центра равна $1 / 2$

$$
\exp \left[-\left(4 \pi \rho r^{3} / 3\right)\right]=1 / 2,
$$

где $\rho$ - концентрация дефектов. В результате получаем среднее расстояние $R=0.55 \rho^{-1 / 3}$. При концентрациях $\rho\left(\mathrm{NV}^{-}\right) \approx 2-3 \mathrm{ppm}$ и $\rho\left(\mathrm{N}^{0}\right) \approx 50 \mathrm{ppm}$ среднее расстояние между двумя соседними NV-центрам составляет 7-8 nm, а расстояние между $\mathrm{NV}$-центром и соседним $\mathrm{N}^{0}$-центром $R\left(\mathrm{NV}-\mathrm{N}^{0}\right) \approx 3 \mathrm{~nm}$. Спектры ОДМР $\mathrm{NV}-\mathrm{N}^{0}$ пар представлены на рис. 2. Видно, что для приведенных оценочных расстояний в этих парах (примерно $3 \mathrm{~nm}$ )

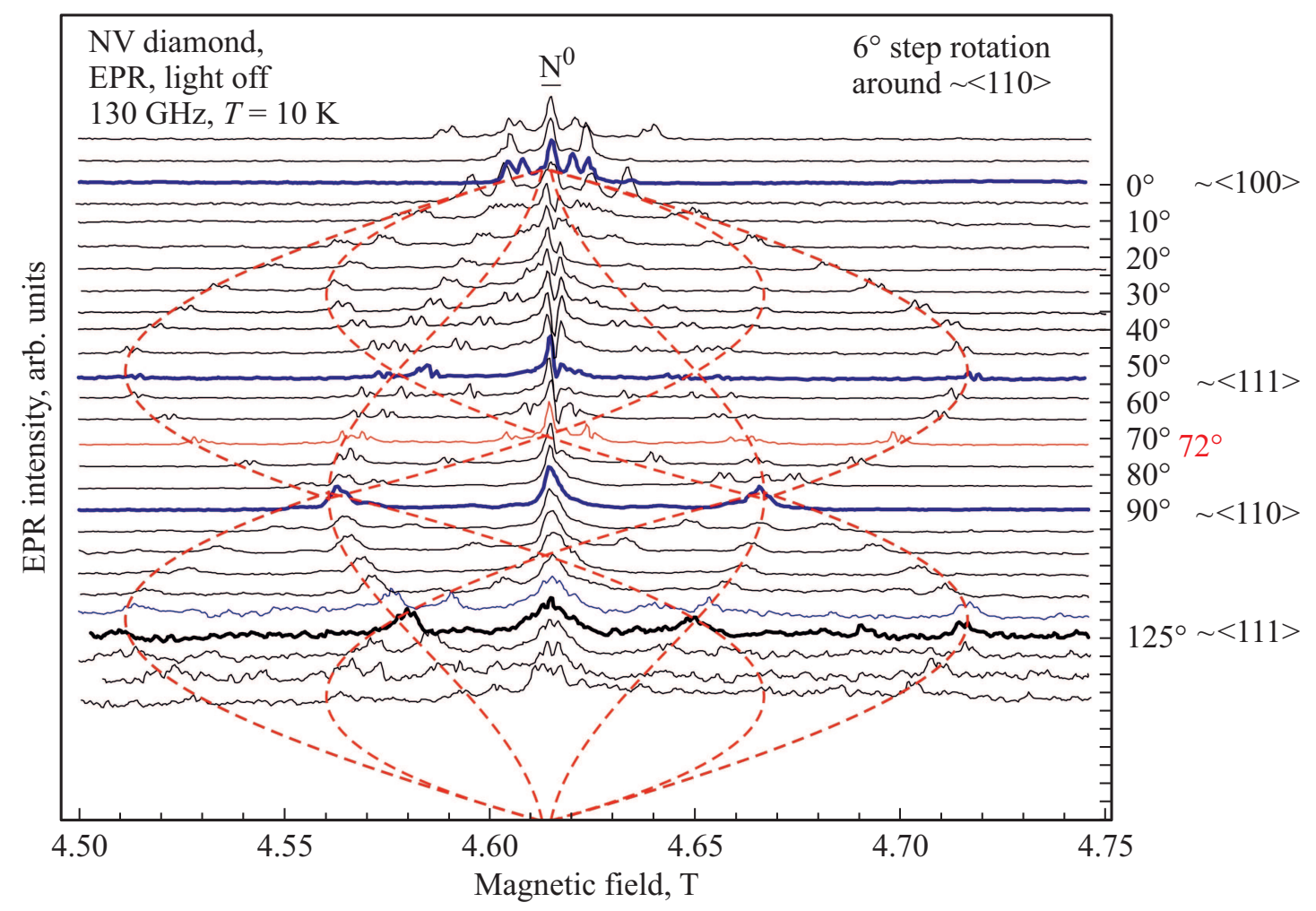

Рис. 4. Ориентационная зависимость спектров ЭПР, зарегистрированных в непрерывном режиме на частоте $130 \mathrm{GHz}$ при температуре $10 \mathrm{~K}$ в монокристалле алмаза, содержащем NV-центры и глубокие доноры атомов азота $\mathrm{N}^{0}$. Вращение образца осуществлялось вокруг оси $\langle 110\rangle$, совпадающей с ребром кристалла, отклонение от оси $\langle 110\rangle$ составляло несколько градусов. Спектры зарегистрированы без оптического возбуждения, то есть в условиях распределения Больцмана для населенностей спиновых уровней в магнитном поле. Частота низкочастотной модуляции магнитного поля составляла $680 \mathrm{~Hz}$, амплитуда модуляции $\sim 0.1 \mathrm{mT}$. Пунктирными линиями обозначены теоретические зависимости положения сигналов ЭПР NV-центров с учетом четырех магнитно-неэквивалентных ориентаций NV-центров вдоль осей $\langle 111\rangle$. 


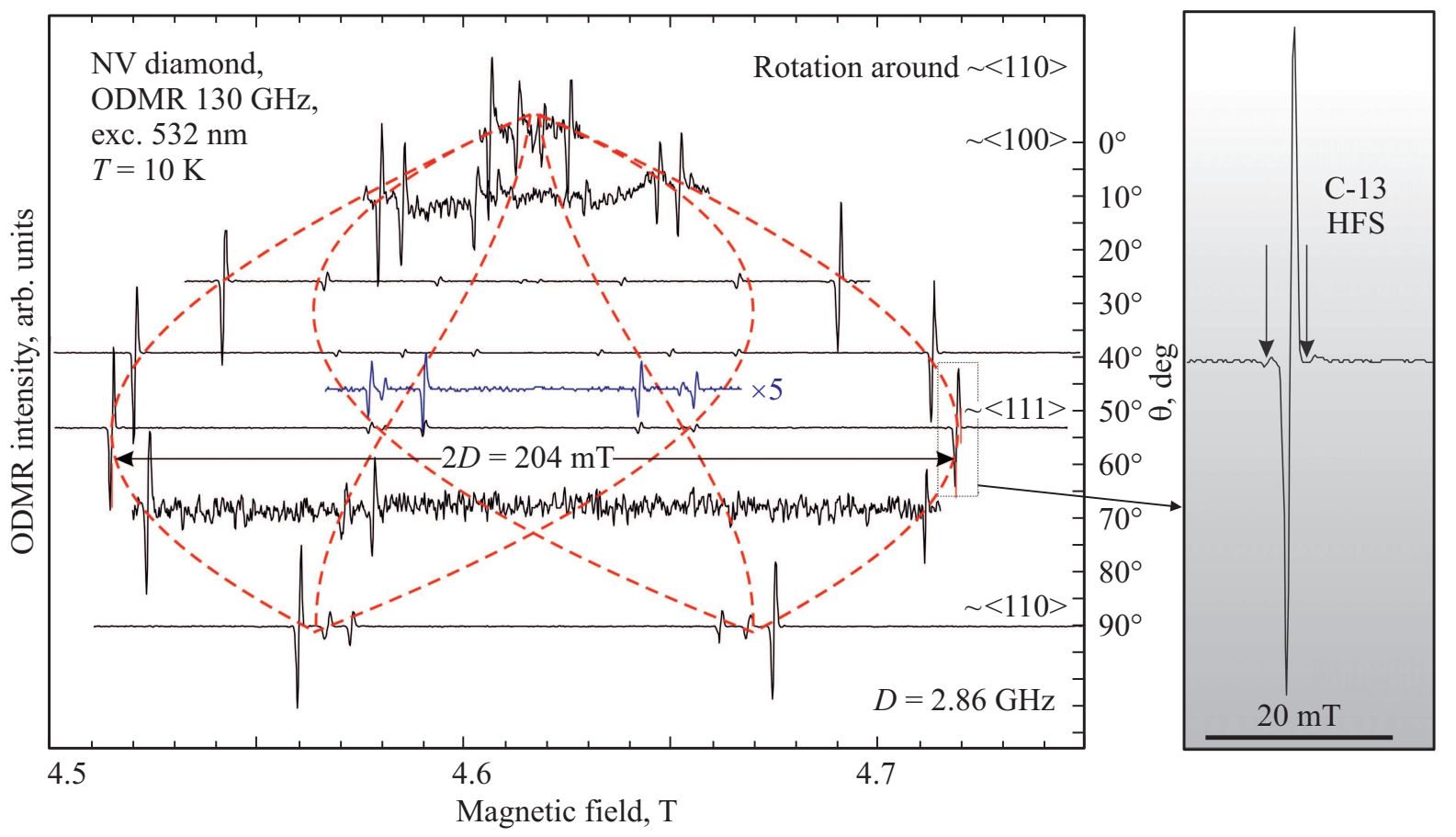

Рис. 5. (а) Ориентационная зависимость спектров ОДМР, зарегистрированных на частоте $130 \mathrm{GHz}$ при температуре $10 \mathrm{~K}$ в монокристалле алмаза, содержащем NV-центры. Вращение осуществлялось вокруг оси $\langle 110\rangle$, совпадающей с ребром кристалла, отклонение от оси $\langle 110\rangle$ составляло несколько градусов. Низкочастотная модуляции магнитного поля осуществлялась на частоте $680 \mathrm{~Hz}$, амплитуда модуляции $\sim 0.1 \mathrm{mT}$. Пунктирными линиями обозначены теоретические зависимости положения сигналов ОДMP NV-центров, с учетом четырех магнитно-неэквивалентных ориентаций NV-центров вдоль осей $\langle 111\rangle$. Фотовозбуждение осуществлялось лазером с длиной волны $532 \mathrm{~nm}$. На вставке - высокопольная линия ОДМР NV-центров с ориентаций магнитного поля вдоль оси 〈111〉 NV-центра, представленная в увеличенном масштабе, позволяющем идентифицировать сверхтонкие компоненты взаимодействия неспаренных электронов с ядром изотопа ${ }^{13} \mathrm{C}$, находящемся в позиции одного из трех ближайших атомов углерода, показанных на рис. 3.

взаимодействия между $\mathrm{NV}$ - и $\mathrm{N}^{0}$-центрами достаточно сильное, чтобы привести к интенсивным сателитам в спектрах ОДМР, отношение интенсивности центральной линии к интенсивности сателлита равно примерно 6.5. Мы считаем, что возможно проведение калибровки отношения амплитуды центральной линии ОДМР к амплитуде сателлитов для определения расстояния между $\mathrm{NV}$-центром и донором азота $\mathrm{N}^{0}$. Эта информация чрезвычайно важна для создания материалов на основе так называемого NV алмаза (NV diamond), так как непосредственно по спектрам ОДМР позволит определить расстояние между NV-центром и донором азота, что определяет когерентные спиновые свойства NV-центров.

На рис. 4 показана ориентационная зависимость спектров ЭПР, зарегистрированных в непрерывном режиме в диапазоне $130 \mathrm{GHz}$ при температуре $10 \mathrm{~K}$ в монокристалле алмаза, содержащем NV-центры и глубокие доноры азота $\mathrm{N}^{0}$ (спектры ОДМР этого кристалла в нулевом магнитном поле представлены на рис. 2). Вращение образца осуществлялось вокруг оси, близкой к направлению $\langle 110\rangle$, совпадающему с ребром кристалла. Отклонение от оси вращения $\langle 110\rangle$ составляло несколько градусов. Спектры зарегистрированы без оптического возбуждения, т. е. в условиях распределения Больцмана для населенностей спиновых уровней, поэтому нарушается симметрия интенсивностей сигналов ЭПР относительно $g \approx 2.0$. Частота никочастотной модуляции магнитного поля составляла $680 \mathrm{~Hz}$, амплитуда модуляции - $0.1 \mathrm{mT}$. Пунктирными линиями обозначены теоретические зависимости положения сигналов ЭПР NV-центров, с учетом четырех магнитно-неэквивалентных ориентаций NV-центров вдоль осей $\langle 111\rangle$. Двойные линии на экспериментальных спектрах обусловлены как неточностью установки ориентации, так и возможным влиянием большого размера кристалла сравнимого с рабочей длиной волны $2 \mathrm{~mm}(130 \mathrm{GHz})$. Эти расщепления полностью исчезают в спектрах ОДМР, зарегистрированных при тех же условиях вращения кристалла, поскольку в ОДМР выделяется малая область кристалла с субмилиметровыми размерами.

На рис. 5, a показана ориентационная зависимость спектров ОДМР, зарегистрированных на частоте $130 \mathrm{GHz}$ при температуре $10 \mathrm{~K}$ в том же монокристалле алмаза, что и на рис. 4, содержащем NV-центры. Фотолюминесценция возбуждалась лазером с длиной волны $532 \mathrm{~nm}$. Вращение образца в магнитном поле также осуществлялось вокруг оси, отклонение от $\langle 110\rangle$ составляло несколько градусов. Частота низкочастот- 
ной модуляции магнитного поля $680 \mathrm{~Hz}$, амплитуда модуляции $\sim 0.1 \mathrm{mT}$. Пунктирными линиями обозначены теоретические зависимости положения сигналов ОДМР $\mathrm{NV}$-центров, с учетом четырех магнитно-неэквивалентных ориентаций NV-центров вдоль осей $\langle 111\rangle$. На вставке показана высокопольная линия ОДМР NV-центров с ориентаций магнитного поля вдоль оси $\langle 111\rangle$, представленная в увеличенном масштабе. Стрелками отмечены две сверхтонкие компоненты, обусловленные взаимодействием неспаренных электронов с одним ядром изотопа ${ }^{13} \mathrm{C}$, находящемся в позиции одного из трех ближайших атомов углерода, показанных на рис. 3. Направление на эти атомы углерода от центра вакансии углерода составляют $70^{\circ}$ с осью выбранного NV-центра. Имеются литературные данные по величинам упомянутых сверхтонких взаимодействий: $A_{\|}=198 \mathrm{MHz}(70.8 \mathrm{G})$; $A_{\perp}=121 \mathrm{MHz}(43.2 \mathrm{G}) ; A\left(70^{\circ}\right)=132 \mathrm{MHz}(47.2 \mathrm{G})$. Beроятность нахождения изотопа ${ }^{13} \mathrm{C}$ в ближайшем окружении вакансии повышается в три раза, поскольку все три атома углерода для рассматриваемой ориентации являются эквивалентными. Природное содержание изотопа ${ }^{13} \mathrm{C}$ составляет $1.1 \%$, т.е. сотношение интенсивностей центральной линии к сателитам теоретически составляет 66. В ОДМР, как правило, относительная амплитуда сверхтонких сателитов несколько возрастает по сравнению со спектрами традиционного ЭПР.

Рассмотрим спектр ОДМР в ориентации, когда магнитное поле направлено вдоль оси $\langle 111\rangle$, совпадающей с осью симметрии одного из четырех NV-центров, для трех других центров ориентации составляют примерно $70^{\circ}$. В идеальном случае сигналы ОДМР от этих трех NV-центров совпадают, и наблюдается только пара линий (см. теоретические зависимости), в реальном случае нашего эксперимента из-за неточности установки ориентации кристалла спектры ОДМР расщепляются на несколько пар линий. Теоретически интенсивности всех линий ЭПР не должны меняться с изменением ориентации магнитного поля в кристалле, однако наши эксперименты показывают, что интенсивности линий ОДМР для ориентации $\theta=0^{\circ}$, намного выше, чем интенсивности линий ОДМР для $\theta \approx 70^{\circ}$. Этот результат может быть обусловлен зависимостью динамических процессов, происходящих при регистрации спектров ОДМР, от ориентации магнитного поля относительно оси симметрии NV-центров. Эти переходные процессы включают динамику оптически-индуцированного выстраивания населенностей спиновых уровней в больших магнитных полях, при этом населяется уровень $M_{S}=0$ и опустошаются уровни $M_{S}= \pm 1$. Резонансное поглощение (излучение) высокочастотных квантов между уровнем $M_{S}=0$ и уровнями $M_{S}= \pm 1$ изменяет населенности спиновых уровней, что, в свою очередь, приводит к сильному (до $10 \%$ ) изменению интенсивности фотолюминесценции в момент магнитного резонанса. Таким образом, отклонение магнитного поля от оси NV-центра приводит к существенному уменьшению изменения интенсивности фотолюминесценции в момент магнитного резонанса,

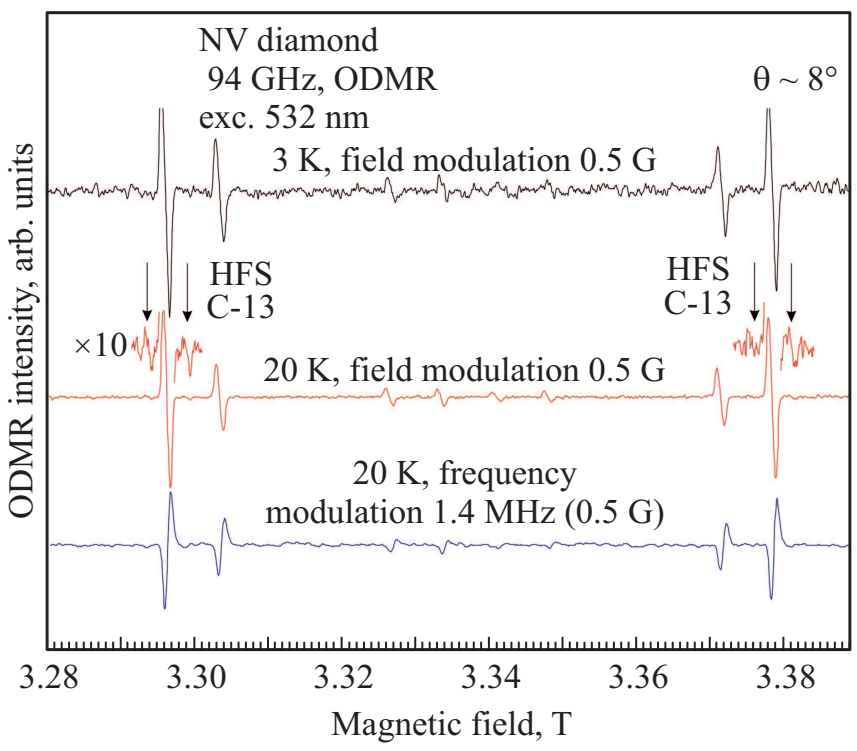

Рис. 6. Спектры ОДМР, зарегистрированные на частоте $94 \mathrm{GHz}$ в монокристалле алмаза, содержащм NV-центры, в ориентации магнитного поля относительно оси с кристалла $\theta=8^{\circ}$ (близкой к ориентации $\langle 100\rangle$ ) при температурах 3 и $20 \mathrm{~K}$ в условиях низкочастотной модуляции магнитного поля с амлитудой $0.05 \mathrm{mT}$. Внизу показан спектр ОДМР, зарегистированный с низкочастотной модуляцией рабочей частоты $94 \mathrm{GHz}$ с амплитудой $1.4 \mathrm{MHz}$, сответствующей амплитуде модуляции магнитного поля $0.05 \mathrm{mT}(1 \mathrm{mT}$ соответствует $28 \mathrm{MHz})$. Частота никочастотной модуляции магнитного поля и рабочей частоты составляла $680 \mathrm{~Hz}$. Фотовозбуждение осуществлялось лазером с длиной волны $532 \mathrm{~nm}$.

что следует учитывать при работе с NV-центрами в сильных магнитных полях. Одной из возможных причин уменьшения интенсивности сигнала ОДМР является зависимость спин-решеточной релаксации от ориентации магнитного поля.

Рис. 6 показывает спектры ОДМР, зарегистрированные на частоте $94 \mathrm{GHz}$ в монокристалле алмаза, содержащего NV-центры, при ориентации магнитного поля относительно оси NV-центра кристалла $\theta \sim 8^{\circ}$ (близкой к ориентации $\langle 100\rangle$ монокристалла алмаза). Фотовозбуждение осуществлялось лазером с длиной волны $532 \mathrm{~nm}$. Спектры измерены при температурах 3 и $20 \mathrm{~K}$ в условиях низкочастотной модуляции магнитного поля с амлитудой $\sim 0.05 \mathrm{mT}$. Внизу показан спектр ОДМР, зарегистированный с низкочастотной модуляцией рабочей частоты $94 \mathrm{GHz}$ с амплитудой $1.4 \mathrm{MHz}$, сответствующей амплитуде модуляции магнитного поля $0.05 \mathrm{mT}$ ( $1 \mathrm{mT}$ соответствует $28 \mathrm{MHz}$ ) [23]. Частота модуляции магнитного поля и рабочей частоты составляла 680 Гц. Наблюдаются сигналы ОДМР NV-центров, с учетом четырех магнитнонеэквивалентных ориентаций NV-центров вдоль осей $\langle 111\rangle$. В этом эксперименте впервые продемонстрирована новая модуляционная методика регистрации спектров ОДМР NV-центров с низкочастотной модуляцией мик- 


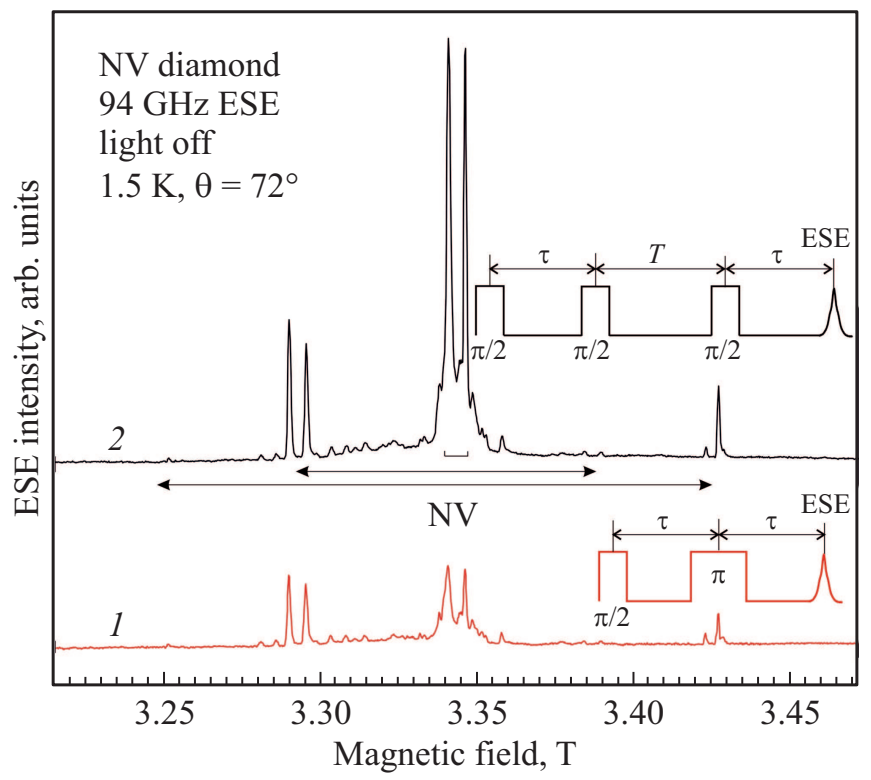

Рис. 7. Спектры электронного спинового эха, зарегистрированные на частоте $94 \mathrm{GHz}$ в монокристалле алмаза, содержащем NV-центры, в ориентации магнитного поля относительно оси с кристалла $\theta=72^{\circ}$ (ориентация между $\langle 111\rangle$ и $\langle 110\rangle$, см. рис. 2) при температуре $1.5 \mathrm{~K}$. Использовались стандартная двухимпульсная схема $\pi / 2=28.8 \mathrm{Hc}, \tau=211.2 \mathrm{~ns}$, $\pi=57.6 \mathrm{~ns} \quad(1)$ и трехимпульсная схема $\pi / 2=28.8 \mathrm{~ns}$, $\tau=211.2 \mathrm{~ns}, \pi / 2=28.8 \mathrm{~ns}, T=400 \mathrm{~nm}, \pi / 2=28.8 \mathrm{~ns}$ (2). Наблюдаются сигналы ОДМР NV-центров, с учетом четырех магнитно-неэквивалентных ориентаций NV-центров вдоль осей $\langle 111\rangle$.

роволновой частоты. Такая методика не требует использования модуляционных катушек, которые в сильных магнитных полях создают дополнительные шумы из-за вибраций.

На рис. 7 представлены спектры электронного спинового эха, зарегистрированные на частоте $94 \mathrm{GHz}$ в монокристалле алмаза, содержащем NV-центры, в ориентации магнитного поля относительно оси с кристалла $\theta \approx 72^{\circ}$ (ориентация между $\langle 111\rangle$ и $\langle 110\rangle$, см. рис. 4) при температуре $1.5 \mathrm{~K}$. Использовались стандартные двух- (1) и трехимпульсная последовательности (2) [24]. Наблюдается несколько линий ЭСЭ NV-центров в связи c наличием четырех магнитно-неэквивалентных ориентаций NV-центров вдоль осей $\langle 111\rangle$ (см. рис. 4). В области $g=2$ регистрируется также сигнал доноров азота. Отметим, что при использовании трех-импульсной схемы регистрации интенсивность сигнала доноров азота увеличивается. Так как оптическое возбуждение отсутствует, интенсивности сигналов NV-центров определяются распределением Больцмана, при этом, в зависимости от угла между осью центра и магнитным полем, большую интенсивность имеет либо высокопольная линия, либо низкопольная с учетом четырех магнитнонеэквивалентных NV-центров. Наблюдается также серия линий ЭСЭ малой интенсивности в диапазоне магнит- ных полей 3.28-3.32 Т, идентификация которых требует дополнительных исследований.

\section{4. Заключение}

Продемонстрированы возможности многоцелевого высокочастотного спектрометра магнитного резонанса при исследовании алмаза, содержащего NV-центры: зарегистрирован высокочастотный ЭПР NV-центров в непрерывном и импульсном режимах, одновременно получены спектры ОДМР с возможностью регистрации сигнала магнитного резонанса с синхронным детектированием на низкой частоте с модуляцией магнитного поля и модуляцией рабочей частоты. Зарегистрированы спектры ЭСЭ с использованием двухимпульсной и трехимпульсных схем. В спектрах ОДМР получены сигналы на сверхтонких компонентах на ядрах углерода ${ }^{13} \mathrm{C}$, что потенциально позволяет изучать оптически ЯМР, а также получать информацию о возможной поляризации ядер при насыщении ядерных переходов на двойных частотах. Показано, что в условиях сильных магнитных полей и широком диапазоне температур, сохраняются все процессы оптически-индуцированного выстраивания спиновых уровней и резонансного микроволнового воздействия на интенсивность ФЛ, наблюдавшиеся ранее для нулевых и малых магнитных полей. Наблюдение сильных ОДМР сигналов в высоких магнитных полях, отличающихся чрезвычайно узкими линиями, дают возможность использовать эти линии, как и в случае малых магнитных полей, для измерения магнитных полей и их пространственного распределения. Обнаружено влияние ориентации сильного магнитного поля относительно оси NV-центра на интенсивность сигнала ОДМР в пределах одного порядка, при этом максимальный ОДМР сигнал регистрируется для ориентации магнитного поля вдоль оси NV-центра.

\section{Финансирование работы}

Работа поддержана РФФИ, грант № 19-52-12058 и ДФГ, Deutsche Forschungsgemeinschaft (DFG) в рамках ICRC project TRR 160.

\section{Конфликт интересов}

Авторы заявляют, что у них нет конфликта интересов.

\section{Список литературы}

[1] G. Davies, M.F. Hamer. Proc. R. Soc. Lond. A 348, 285 (1976).

[2] A. Gruber, A. Dŕabenstedt, C. Tietz, L. Fleury, J. Wrachtrup, C. von Borczyskowski. Science 276, 2012 (1997).

[3] F. Jelezko, I. Popa, A. Gruber, C. Tietz, J. Wrachtrup, A. Nizovtsev, S. Kilin. Appl. Phys. Lett. 81, 2160 (2002).

[4] A.P. Nizovtsev, S.Ya. Kilin, F. Jelezko, I. Popa, A. Gruber, J. Wrachtrup. Phys. B: Condens. Matter 340-342, 106 (2003). 
[5] J.R. Maze, P.L. Stanwix, J.S. Hodges, S. Hong, J.M. Taylor, P. Cappellaro, L. Jiang, M.V. Gurudev Dutt, E. Togan, A.S. Zibrov, A. Yacoby, R.L. Walsworth, M.D. Lukin. Nature 455, 644 (2008).

[6] J.M. Taylor, P. Cappellaro, L. Childress, L. Jiang, D. Budker, P.R. Hemmer, A. Yacoby, R. Walsworth, M.D. Lukin. Nature Phys. 4, 810 (2008).

[7] G. Balasubramanian, I.Y. Chan, R. Kolesov, M. Al-Hmoud, J. Tisler, C. Shin, C. Kim, A. Wojcik, P.R. Hemmer, A. Krueger, T. Hanke, A. Leitenstorfer, R. Bratschitsch, F. Jelezko, J. Wrachtrup. Nature Lett. 455, 648 (2008).

[8] P.-H. Chung, E. Perevedentseva, J.-S. Tu, C.C. Chang, C.-L. Cheng. Diamond Rel.Mater. 15, 622 (2006).

[9] Y.-R. Chang, H.-Y. Lee, K. Chen, C.-C. Chang, D.-S. Tsai, C.-C. Fu, T.-S. Lim, Y.-K. Tzeng, C.-Y. Fang, C.-C. Han, H.-C. Chang, W. Fann. Nature Nanotechnol. 3, 284 (2008).

[10] S.J. Yu, M.W. Kang, H.C. Chang, K.M. Chen, Y.C. Yu. J. Am. Chem. Soc. 127, 17604 (2005).

[11] T.M. Babinec, B.J.M. Hausmann, M. Khan, Y. Zhang, J.R. Maze, P.R. Hemmer, M. Loncar. Nature Nanotechnol. 5, 195 (2010).

[12] P.G. Baranov, A.A. Soltamova, D.O. Tolmachev, N.G. Romanov, R.A. Babunts, F.M. Shakhov, S.V. Kidalov, A.Y. Vul', G.V. Mamin, S.B. Orlinskii, N.I. Silkin. Small 7, 1533 (2011).

[13] J. Wrachtrup, F. Jelezko. J. Phys.: Condens. Matter 18, 807 (2006).

[14] Е.В. Единач, Ю.А. Успенская, А.С. Гурин, Р.А. Бабунц, Х.Р. Асатрян, Н.Г. Романов, А.Г. Бадалян, П.Г. Баранов. ФTT 61, 1864 (2019) [Phys. Solid State 61, 10 (2019)].

[15] E.V. Edinach, Yu.A. Uspenskaya, A.S. Gurin, R.A. Babunts, H.R. Asatryan, N.G. Romanov, A.G. Badalyan, P.G. Baranov. Phys. Rev. B 100, 104435 (2019).

[16] Р.А. Бабунц, А.Г. Бадалян, Е.В. Единач, А.С. Гурин, Н.Г. Романов, П.Г. Баранов. Высокочастотный спектрометр электронного парамагнитного резонанса. Патент РФ № 2711345 от 16 января 2020 г.

[17] S. Felton, A.M. Edmonds, M.E. Newton, P.M. Martineau, D. Fisher, D.J. Twitchen, J.M. Bake. Phys. Rev. B 79, 075203 (2009).

[18] R.A. Babunts, A.A. Soltamova, D.O. Tolmachev, V.A. Soltamov, A.S. Gurin, A.N. Anisimov, V.L. Preobrazhenskii, P.G. Baranov. JETP Lett. 95, 429 (2012).

[19] V.M. Acosta, E. Bauch, M.P. Ledbetter, A. Waxman, L.S. Bouchard, D. Budker, Phys. Rev. Lett. 104, 070801 (2010).

[20] P. Neumann, I. Jakobi, F. Dolde, C. Burk, R. Reuter, Waldherr, J. Honert, T. Wolf, A. Brunner, J.H. Shim, D. Suter, H. Sumiya, J. Isoya, J. Wrachtrup. Nano Lett. 13, 2738 (2013).

[21] P.G. Baranov, H.J. von Bardeleben, F. Jelezko, J. Wrachtrup. Magnetic Resonance of Semiconductors and Their Nanostructures: Basic and Advanced Applications: Ser. Mater. Sci. Springer. V. 253. (2017).

[22] M.J.R. Hoch, E.C. Reynhardt. Nuclear-spin-lattice relaxation of dilute spins in semiconducting diamond. Phys. Rev. B 37, 9222 (1988).

[23] Р.А. Бабунц, А.Г. Бадалян, Ю.А. Успенская, А.С. Гурин, Н.Г. Романов, П.Г. Баранов. Высокочастотный спектрометр электронного парамагнитного резонанса. Патент РФ № 2711228 от 15 января 2020 г.

[24] A. Schweiger, G. Jeschke. Principles of Pulse Electron Paramagnetic Resonance. Oxford University Press (2001).

Редактор Е.В.Емцев 